\title{
Mineração
}

\section{Fundamentos para simulação dos desmontes de rocha por explosivos}

\author{
Juarez Lopes de Morais \\ Doutorando em Engenharia Mineral pela UFMG, \\ Engenheiro Master da Companhia Vale do Rio Doce (Mina de cobre do Sossego) \\ E-mail: juarez.morais@cvrd.com.br \\ Maria de Fátima Andrade Gripp \\ Eng. de Minas, Dr. Ing. ENSMP, \\ Professor Adjunto Escola de Engenharia UFMG \\ E-mail:mgripp@demin.ufmg.br
}

\section{Resumo}

Esse artigo apresenta alguns resultados do trabalho de pesquisa realizado para preparação da tese de doutorado sobre a utilização da simulação da fragmentação no processo de desmonte de rochas por explosivos. É apresentado um programa de computador denominado SIMBLAST que utiliza a linguagem de programação Visual Basic. O programa foi desenvolvido com base nos modelos Kuz-Ram e TCM - Two Components Model, descritos nesse artigo. Os dados para teste do simulador foram levantados nas detonações de hematita dura (HD) e hematita mole (HM), nas minas de minério de ferro de Carajás, da Companhia Vale do Rio Doce, localizadas no sul do Estado do Pará, com a finalidade de comparação dos resultados da curva granulométrica gerada pelos dois modelos.

Palavras-chave: Simulação da fragmentação, desmonte de rochas, explosivos, Kuz-Ram, TCM.

\begin{abstract}
This article shows some results of the doutorate thesis about blast simulation. It's looked for the development of blast simulation estabilished in KuzRam and TCM (Two Components Model) models. For that was developed a software called SIMBLAST. The data for this test of simulate was took in the iron mine in Carajás owned by CVRD - Companhia Vale do Rio Doce, located in the south of Para State. The simulator was tested firstly in blasting of hard hematite (HD) and soft hematite (HM) with the intention of compararison of the models
\end{abstract}

Keywords: Blast simulation, explosives, fragmentation, Kuz-Ram, TCM. 


\section{Introdução}

Vários autores têm estudado modelos de interação explosivo-rocha, que fornecem uma descrição útil do processo de detonação como um "background" para modelar o processo de fragmentação da rocha por explosivos. Entre os principais trabalhos destacam-se: Kuznetsov (1973), Cunningham (1983), Lilly (1986), Sarma (1994) e Djordjevic (1999).

Esse artigo apresenta um resumo dos resultados dos trabalhos desses pesquisadores, que estudaram e construíram modelos para a previsão da fragmentação do desmonte a explosivo. Esses modelos foram reunidos para possibilitar a construção de um programa de computador para simulação da fragmentação. A finalidade principal da simulação é a previsão da curva granulométrica da pilha detonada. Isto possibilita a alteração dos parâmetros dos planos de fogo de forma a atingir a fragmentação desejada para cada detonação.

\section{Descrição dos modelos}

As principais equações que compõem os dois modelos de simulação em questão são apresentadas a seguir.

\subsection{O modelo Kuz-Ram}

As propriedades das rochas, as propriedades dos explosivos e as variáveis geométricas do plano de fogo são combinadas usando cinco equações que compõem o modelo de fragmentação Kuz-Ram:

\section{- Equação de Kuznetsov}

Uma correlação entre o tamanho médio do fragmento e a energia de detonação aplicada por unidade de volume de rocha (razão de carga) foi desenvolvida por Kuznetsov (1973) como uma função do tipo de rocha. Essa equação foi modificada por Cunningham (1983) e é dada por:

$X_{50}=A \times(K)^{-0,80} \times Q_{e}^{0,167} \times\left(\frac{115}{E r}\right)^{0,63}$ onde:

$X_{50}$ é o tamanho médio de partícula $(\mathrm{cm})$.

$A$ é o fator da rocha.

$K$ é a razão de carga $\left(\mathrm{kg} / \mathrm{m}^{3}\right)$.

$Q_{e}$ é a massa do explosivo utilizado $(\mathrm{kg})$.

Er representa a energia relativa em massa (RWS) do explosivo comparada ao ANFO $(\mathrm{ANFO}=100)$.

\section{- Equação de Rosin-Rammler:}

A distribuição de tamanho dos fragmentos é calculada pela equação de RosinRammler:

$P=100 \times\left[1-e^{-0,693 \times\left(X / X_{50}\right)^{n}}\right]$

onde:

$X$ é o tamanho da malha da peneira.

$X_{50}$ é o tamanho médio de partícula, n é o índice de uniformidade.

$P$ é o percentual de material passante na peneira de tamanho $\mathrm{X}$.

\section{- Índice de uniformidade de Cunningham:}

Essa expressão foi desenvolvida através de testes de campo por Cunningham (1987). Ela correlaciona todos os parâmetros geométricos do plano de fogo, como segue:

$$
n=\left[2,2-14 \times\left(\frac{B}{D}\right)\right] \times\left[\frac{(1+S / B)}{2}\right]^{0,5} \times\left\{\left(1-\frac{W}{B}\right) \times\left[a b s \times \frac{(B C L-C C L)}{L}+0,1\right]^{0,1} \times\left(\frac{L}{H}\right)\right\}
$$

onde:

$B$ é o afastamento (m).

$S$ é o espaçamento $(\mathrm{m})$.

$D$ é o diâmetro do furo $(\mathrm{mm})$.

$W$ é o desvio da perfuração (m).

$L$ é o comprimento total de carga $(\mathrm{m})$.

$H$ é a altura do banco (m).

$B C L$ é o comprimento da carga de fundo (m).

$C C L$ é o comprimento da carga de coluna (m).

$a b s$ é o valor absoluto referente a (BCL-CCL)/L.

\section{-Equação de Tidman:}

A energia do explosivo é calculada a partir da equação desenvolvida por Tidman:

$E r=\left(\frac{V O D_{e}}{V O D_{n}}\right)^{2} \times R W S$

onde:

$E r$ é a energia relativa por massa efetiva do explosivo.

$V O D_{e}$ é a velocidade de detonação efetiva do explosivo (medida em campo).

$V O D_{n}$ é a velocidade de detonação nominal do explosivo $(\mathrm{m} / \mathrm{s})$.

$R W S$ representa a energia relativa por massa comparada ao ANFO.

\section{- Fator da rocha:}

O fator da rocha (A) foi originalmente desenvolvido por Lilly (1986) e modifica- 
da por Cunningham (1987). Para determinação desse fator são usados os dados obtidos na classificação da Tabela 1 , a qual considera o tipo de rocha, direção e mergulho das descontinuidades com relação à frente livre do desmonte. O fator da rocha é usado para ajustar o tamanho médio dos fragmentos do modelo e é obtido por:

$A=0,06 \times(R M D+J F+R D I+H F)$

\subsection{O Modelo TCM}

Para garantir uma maior precisão na previsão da fragmentação obtida no desmonte, principalmente para os finos, foi desenvolvido o modelo de duas componentes (TCM), Djordjevic (1999). Nesse modelo, a pilha resultante do desmonte pode ser considerada uma mistura de dois conjuntos de fragmentos de rocha, ilustrados na Figura 1. O primeiro conjunto

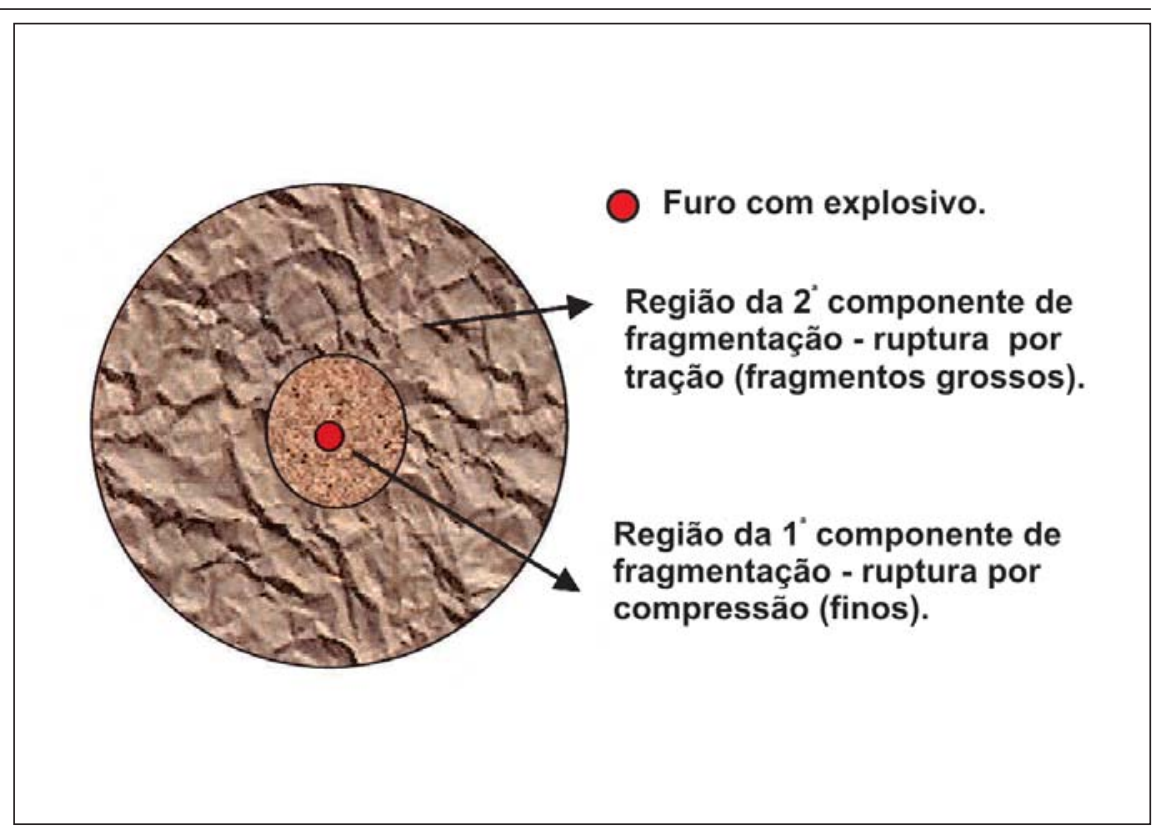

Figura 1 - Modelo de fragmentação TCM, mostrando as duas componentes de fragmentação do maciço rochoso (Djordjevic, 1999).

Tabela 1 - Classificação geomecânica para obtenção do fator da rocha (Cunningham, 1983).

\begin{tabular}{|c|c|c|c|}
\hline Símb. & Descrição & Classificação & Índice \\
\hline \multirow{3}{*}{ RMD } & \multirow{3}{*}{ Maciço rochoso } & Friável & 10 \\
\hline & & Fraturado & JF \\
\hline & & Maciço & 50 \\
\hline JF & Maciço fraturado & JPS + JPA & \\
\hline \multirow{3}{*}{ JPS } & \multirow{3}{*}{ Espaçamento das descontinuidades (m) } & $<0,10 \mathrm{~m}$ & 10 \\
\hline & & 0,10 a MS & 20 \\
\hline & & MS a DP & 50 \\
\hline MS & Oversize da britagem primária $(\mathrm{m})$ & & \\
\hline DP & Parâmetros da malha de perfuração $(\mathrm{m})$ & & \\
\hline \multirow{4}{*}{ JPA } & \multirow{4}{*}{ Direção e mergulho com relação à face livre } & Horizontal & 10 \\
\hline & & Mergulhando para fora da face livre & 20 \\
\hline & & Direção perpendicular à face livre & 30 \\
\hline & & Mergulhando para dentro da face & 40 \\
\hline RDI & $\begin{array}{l}\text { Influência da densidade (densidade da rocha } \\
\text { intacta, } \mathrm{g} / \mathrm{cm}^{3} \text { ) }\end{array}$ & $\mathrm{RDI}=25 \mathrm{~d}-50$ & \\
\hline \multirow{2}{*}{$\mathrm{HF}$} & se $\mathrm{E}<50 \mathrm{GPa}$ & $\mathrm{HF}=\mathrm{E} / 3$ & \\
\hline & se $\mathrm{E}>50 \mathrm{GPa}$ & $\mathrm{HF}=\mathrm{UCS} / 5$ & \\
\hline$E$ & Módulo de Young (GPa) & & \\
\hline UCS & Resistência à compressão uniaxial (MPa) & & \\
\hline
\end{tabular}


origina-se da rocha relativamente próxima ao furo. Esse conjunto de fragmentos tem ruptura compressiva-cisalhante; a influência da estrutura do maciço rochoso tende a ser muito pequena no resultado dessa fragmentação. O segundo conjunto de fragmentos de rocha, tipicamente com granulometria mais grosseira do que o primeiro, é proveniente da rocha mais distante do furo. Esses fragmentos de rocha são criados por ruptura por tração, por meio da abertura e extensão de fraturas preexistentes, planos de acamamento e descontinuidades do maciço rochoso. Esse conjunto de fragmentos abrange uma região muito maior do que a região da primeira componente de fragmentação.

Assumindo que a massa de rocha que se fragmenta devido à ruptura compressiva-cisalhante representa a fração $F$ da massa total de rocha desmontada por furo, tem-se:

$F_{c}=\frac{M_{0}}{M}$

onde:

$M_{o}$ é a massa de rocha fragmentada por compressão/cisalhamento.

$M$ representa a massa total por furo.

Conseqüentemente, a fração de rocha que se rompe por tração ao longo das descontinuidades preexistentes é 1 - Fc. Por causa dos diferentes mecanismos de ruptura, cada subconjunto de fragmentos de rochas precisa ser representado como uma função distinta de distribuição de tamanho. Usando a forma de distribuição de Rosin-Rammler, tem-se:

$$
\begin{aligned}
& P_{1}=100 \times\left[1-e^{-0,693 \times(x / c)^{d}}\right] \\
& P_{2}=100 \times\left[1-e^{-0,693 \times(x / a)^{b}}\right]
\end{aligned}
$$

onde:

$P_{1}$ e $P_{2}$ são os percentuais passantes na peneira de tamanho (x) para as regiões de ruptura por compressão e tração, respectivamente.

$c$ é o tamanho médio de fragmento na primeira região (ruptura por compressão). $d$ representa o coeficiente de uniformidade da primeira distribuição de tamanho de fragmentos. $a$ representa o tamanho médio de fragmento na segunda região (ruptura por tração). $b$ é o coeficiente de uniformidade da segunda distribuição de tamanho de fragmentos.

A soma das duas funções de distribuição, multiplicada pelas respectivas frações da massa total, Fc e (1 - Fc), representará a distribuição de tamanho dos fragmentos da massa total:

$$
P=100 \times\left[1-\left(1-F_{c}\right) \times e^{-0,693 \times(x / a)^{b}}-F_{c} \times e^{-0,693 \times(x / c)^{d}}\right]
$$

A fragmentação fina da rocha é predominantemente controlada pela interação do explosivo com a rocha intacta (rocha matriz) e é gerada na região da primeira componente ilustrada na Figura 1. Nessa região, os fragmentos típicos têm tamanho menor que 50mm. A influência dos aspectos macroestruturais do maciço e dos parâmetros geométricos do plano de fogo (afastamento, espaçamento etc.) não é importante na distribuição granulométrica desses fragmentos.

O campo de tensões na zona de ruptura cisalhante em volta do furo é compressivo. Ambas as tensões, radial e tangencial, são compressivas. Em tal caso, a extensão da ruptura cisalhante em volta do furo pode ser estimada usando o critério de ruptura de Griffith, que, para o caso bi-axial de carregamento predominantemente compressivo, é (Djordjevic, 1999):

$\left(\sigma_{1}-\sigma_{2}\right)^{2}-8 T_{0} \times\left(\sigma_{1}+\sigma_{2}\right)=0$

onde:

$\sigma_{1}$ e $\sigma_{2}$ são as tensões principais maior e menor, respectivamente.

$T_{0}$ é a resistência à tração da rocha.

Assumindo um coeficiente de Poisson( $(v)$ médio de 0,25 e usando $\sigma_{2}=[v /(1-v)] \cdot \sigma_{1}$, a equação para cálculo do raio da região da primeira componente é:

$$
r=0,5 \times \frac{D}{\left(24 T_{0} / P_{b}\right)^{1 / 2}}
$$

onde:

r é o raio da zona de pulverização.

$D$ é o diâmetro do furo.

$T_{0}$ é a resistência à tração da rocha.

$P_{b}$ é o pico da pressão de detonação no furo.

O pico da pressão de detonação no furo é estimado usando a equação de Person, Holmberg e Lee (1994):

$$
P b=0,25 \times \rho \times\left(V O D_{e}\right)^{2}
$$

onde:

$P_{b}$ é a pressão de detonação aplicada à rocha $(\mathrm{Pa})$.

$\rho$ é a densidade do explosivo $\left(\mathrm{kg} / \mathrm{m}^{3}\right)$.

$V O D_{e}$ é a velocidade de detonação efetiva do explosivo $(\mathrm{m} / \mathrm{s})$.

Para o desenvolvimento do programa de simulação foram estudadas algumas variações desse modelo, principalmente no que refere ao cálculo do tamanho médio e no coeficiente de distribuição dos finos do desmonte.

\subsection{0 programa SIMBLAST}

O programa foi desenvolvido com base nos modelos de simulação de fragmentação Kuz-Ram e TCM. O programa SIMBLAST tem as seguintes características técnicas e computacionais: 
- Arquitetura-cliente/servidor com base de dados Oracle.

- Interface gráfica padrão Microsoft.

- Desenvolvimento utilizando I-CASE Visual Basic 4.032 bits.

- Hardware: a instalação-padrão do SIMBLAST é em arquitetura departamental, requerendo, no mínimo, na estação-cliente um PC 486 DX4 $100 \mathrm{MHz}$, RAM 16MB, mouse, monitor SGVA colorido e placa de rede. Servidor de processos de bancos de dados: servidor RISC/Powdwe PC (Unix ou Windows NT), INTEL (Windows NT).

- Software: sistema operacional Windows 95 (na estação-cliente) e sistema operacional Unix ou Windows NT e RDBMS.

A Figura 2 mostra a tela principal de simulação para o modelo TCM com a curva granulométrica gerada a partir de um plano de fogo.

\section{Resultados}

\subsection{Determinação do fator da rocha}

O simulador foi testado nas detonações de minério de ferro das minas da Companhia Vale do Rio Doce, em Carajás-PA. A Figura 3 mostra a frente de lavra de hematita dura (HD) escolhida para teste do simulador. Para a determinação do fator de rocha, foi utilizada a classificação proposta por Cunningham (1987). Essa classificação foi realizada a partir do mapeamento da frente de lavra com o apoio da geologia da mina. A Tabela 2 apresenta a classificação do fator da rocha para a hematita dura.

\subsection{Dados do plano de fogo}

A Tabela 3 mostra os principais parâmetros do plano de fogo utilizados na detonação da HD.

\subsection{Comparação dos modelos Kuz-Ram e TCM}

Após o levantamento dos dados



Figura 2 - Tela principal de simulação para o modelo TCM.

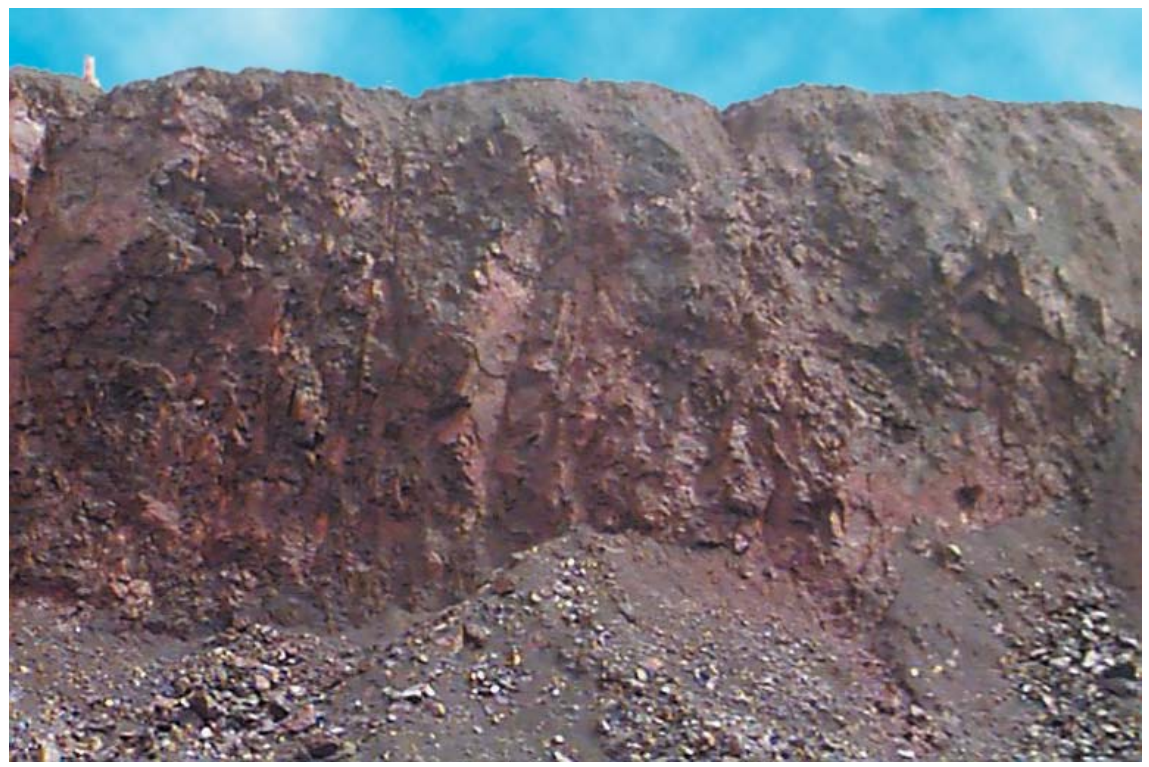

Figura 3 - Vista da frente de lavra da detonação da HD.

geomecânicos da frente de lavra e a entrada dos parâmetros do plano de fogo no simulador SIMBLAST foram geradas as curvas granulométricas para os modelos Kuz-Ram e TCM.

A avaliação dessas curvas mostrou que o modelo TCM apresenta um maior percentual de finos. Já na fração grossa, os dois modelos se equivalem. Isto pode ser comprovado plotando as duas cur- vas numa mesma tela de simulação, como mostra a Figura 4. Nesse caso, abaixo do tamanho de fragmento igual a $20 \mathrm{~cm}$, os modelos TCM e Kuz-Ram apresentam resultados diferentes.

Como a frente de lavra de HD apresentou grande variabilidade nas propriedades do maciço rochoso, foi realizada, também, uma simulação considerando a presença de HM - Hematita Mole na área 
Fundamentos para simulação dos desmontes de rocha por explosivos

Tabela 2 - Determinação do fator da rocha para a HD.

\begin{tabular}{c|c|c|c}
\hline Símb. & Descrição & Classificação & Índice \\
\hline RMD & Maciço rochoso & Fraturada & JF \\
\hline JF & Maciço fraturado & JPS + JPA & 40 \\
\hline JPS & $\begin{array}{c}\text { Espaçamento das } \\
\text { fraturas (m) }\end{array}$ & de 0,1m a 1,0 m & 20 \\
\hline JPA & $\begin{array}{c}\text { Direção e mergulho com } \\
\text { relação à frente livre do } \\
\text { fogo da família principal }\end{array}$ & $\begin{array}{c}\text { Mergulhando para fora da } \\
\text { face livre }\end{array}$ & 20 \\
\hline RDI & Influência da densidade & $25 \times$ d - 50 & 64 \\
\hline d & Densidade (g/cm $\left.{ }^{3}\right)$ & & 4,56 \\
\hline HF & Fator HF & Se/5 & 13,2 \\
\hline Se & $\begin{array}{c}\text { Energia específica de } \\
\text { perfuração média (MPa) }\end{array}$ & & 66 \\
\hline A & Fator da rocha & $\mathrm{A}=0,06 \times(\mathrm{RMD}+\mathrm{JF}+\mathrm{RDI}+\mathrm{HF})$ & 7,03 \\
\hline
\end{tabular}

Tabela 3 - Principais parâmetros do plano de fogo para a HD.

\begin{tabular}{c|c}
\hline Parâmetros & $\begin{array}{c}\text { Plano de } \\
\text { Fogo }\end{array}$ \\
\hline Diâmetro & $121 / 4 "$ \\
\hline Explosivo & $\begin{array}{c}\text { Blendado } \\
60 / 40\end{array}$ \\
\hline Altura do banco $(\mathrm{m})$ & 15 \\
\hline Afastamento $(\mathrm{m})$ & 6,5 \\
\hline Espaçamento $(\mathrm{m})$ & 11,4 \\
\hline Tampão $(\mathrm{m})$ & 5,5 \\
\hline Carga por furo $(\mathrm{kg})$ & 1035 \\
\hline Razão de carga $(\mathrm{g} / \mathrm{t})$ & 204 \\
\hline
\end{tabular}

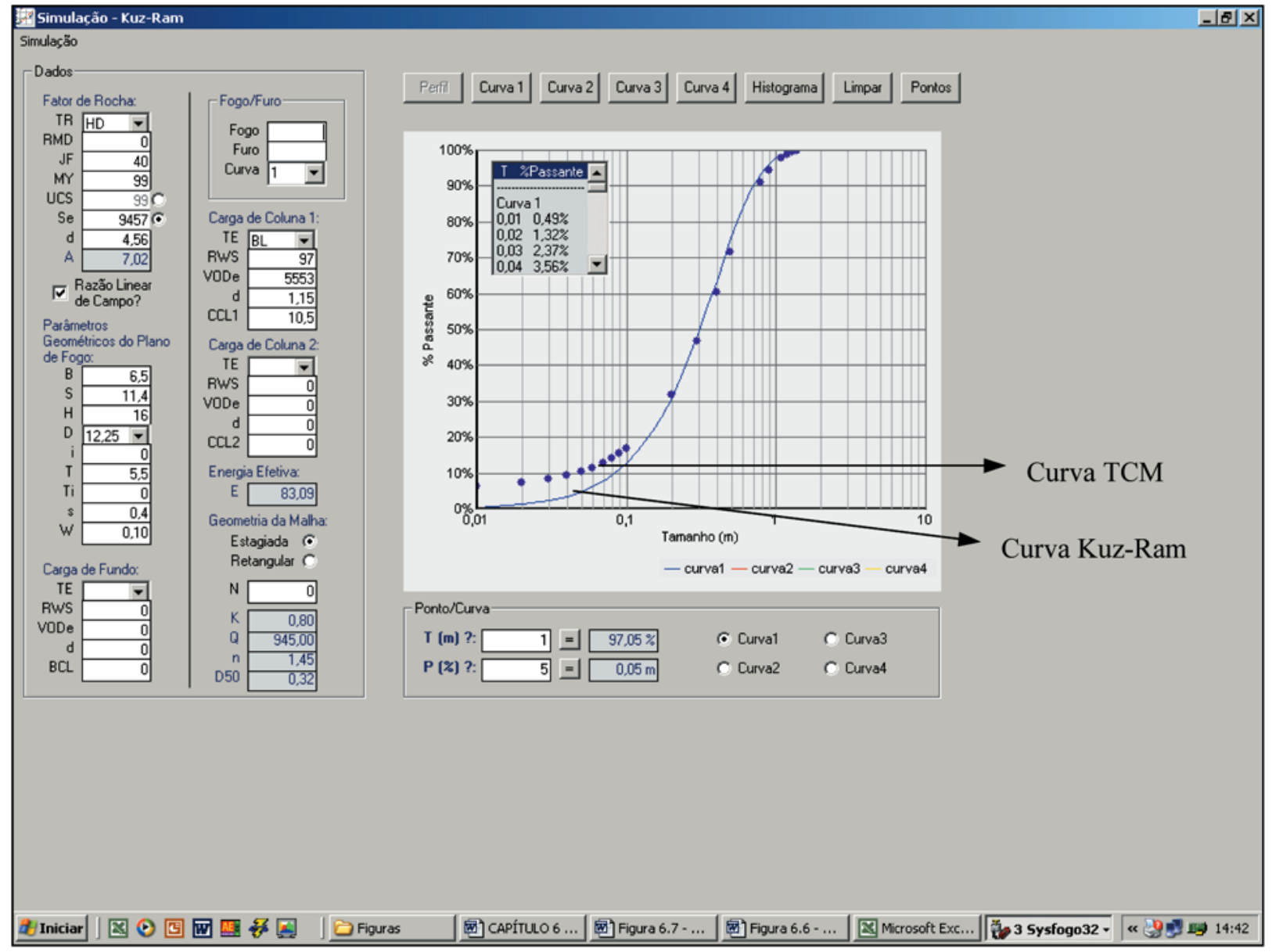

Figura 4 - Comparação das curvas TCM e Kuz-Ram geradas pelo programa SIMBLAST para a detonação da HD. Nota-se nitidamente uma mudança no comportamento das curvas na região onde os fragmentos são menores do que aproximadamente $20 \mathrm{~cm}$. 
Juarez Lopes de Morais et al.

detonada. Para isso, foi recalculado o fator da rocha e realizada uma nova rodada de simulação. Nesse caso, o fator da rocha para a HM reduziu para 2,61 (contra 7,03 na HD). A Figura 5 mostra as novas curvas granulométricas geradas pelos dois modelos para a detonação de HM.

Comparando os resultados da Figura 4 com os resultados da Figura 5, conclui-se que, para maciços rochosos com um menor fator de rocha (menor resistência à compressão, menor espaçamento entre as descontinuidades e menor densidade), ocorre uma maior diferença na região dos finos entre as curvas dos dois modelos. Isto mostra que, para rochas extremamente resistentes e maciças, os modelos Kuz-Ram e TCM tendem apresentar resultados similares.
Segundo Djordjevic (1999), o modelo Kuz-Ram subestima a fração dos finos. No caso das detonações de HD, como o objetivo é a redução da geração de finos, o modelo de simulação que melhor representa a granulometria dos finos deve ser utilizado, nesse caso o TCM.

Os maciços rochosos apresentam, geralmente, grande variabilidade em suas propriedades. Normalmente, não se tem um fogo de produção com apenas um tipo de rocha. Ocorrem intrusões de HM, transições de HM/HD e HD em um mesmo desmonte. Essa situação é bastante complexa para a simulação do desmonte, pois dificulta a determinação do fator da rocha e da curva granulométrica simulada. Um mapeamento detalhado das frentes de lavra permite aumentar a precisão da simulação.

\section{Conclusões}

- O simulador SIMBLAST permite testar várias configurações de plano de fogo antes de sua execução, com maior rapidez e menor custo na definição, adequação e implementação de novos planos de fogo.

- Os modelos Kuz-Ram e TCM apresentam uma diferença na previsão da fração dos finos. Os resultados mostraram uma diferença nas curvas simuladas para fragmentos com tamanho abaixo de $20 \mathrm{~cm}$.

- Para fatores de rocha maiores (rochas mais resistentes), os modelos TCM e Kuz-Ram tendem a apresentar resultados similares de simulação. Já para fatores de rocha menores (rochas menos competente), os modelos apre-

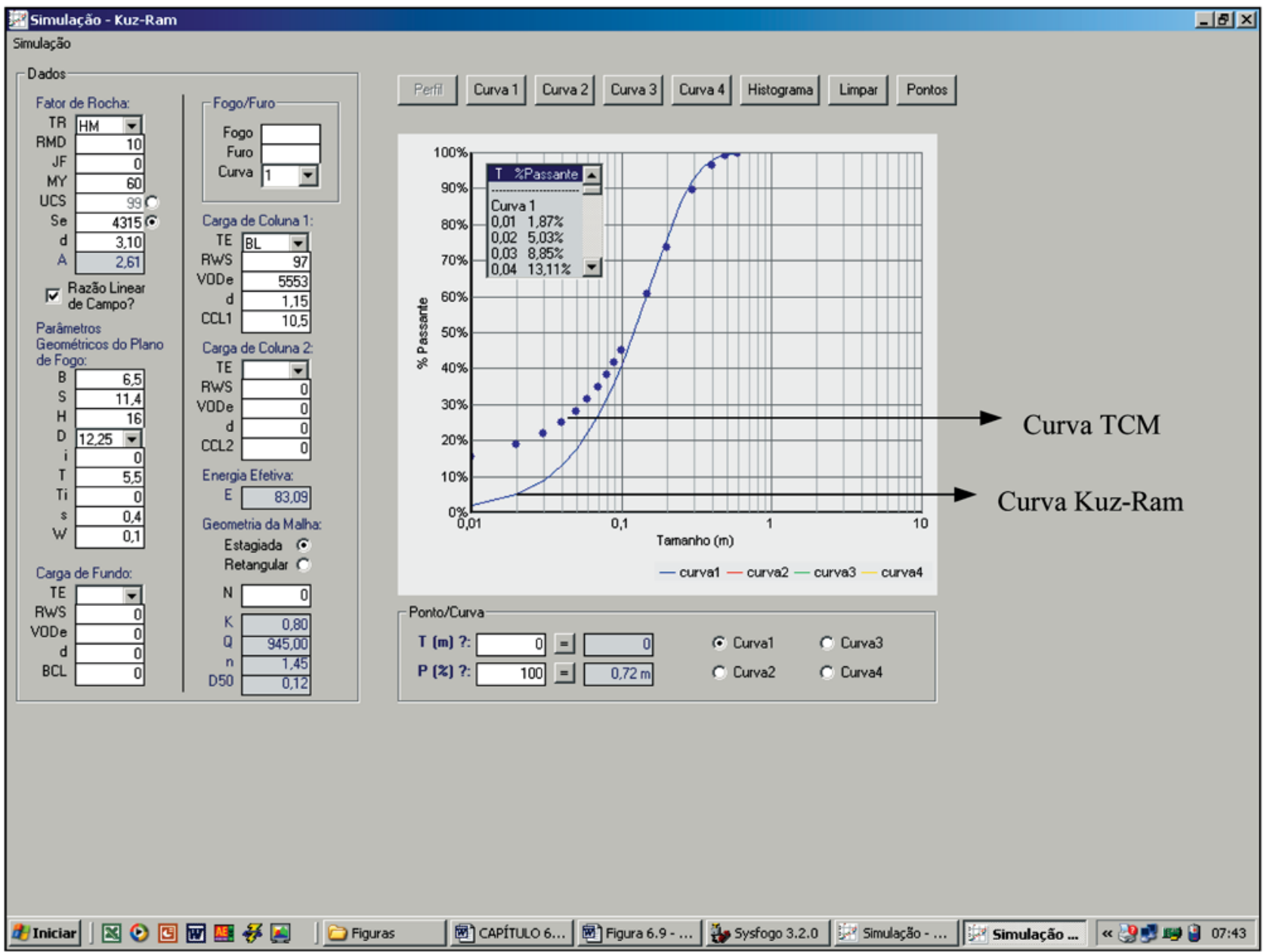

Figura 5 - Comparação das curvas TCM e Kuz-Ram geradas pelo programa SIMBLAST para a detonação de HM. Nota-se nitidamente uma mudança no comportamento das curvas na região onde os fragmentos são menores do que aproximadamente $15 \mathrm{~cm}$. 
sentam maiores diferenças na simulação.

- No modelo TCM é evidente que a geração de finos no desmonte aumenta com o aumento do diâmetro de perfuração e da velocidade de detonação do explosivo.

- As propriedades da rocha intacta relevantes para o projeto de desmonte a explosivo são o módulo de Young, coeficiente de Poisson, resistência à compressão uniaxial e densidade. Essas propriedades, aliadas ao padrão de fraturamento do maciço rochoso, determinam a aptidão ao desmonte do maciço. Esse padrão de fraturamento, estabelecido pela atitude (direção e mergulho) e espaçamento das famílias de descontinuidades e descontinuidades aleatórias, determina o tamanho e forma dos blocos. O mapeamento geomecâmico das frentes de lavra para o desmonte é, por essa razão, de suma importância para a elaboração do plano de fogo.

\section{Referências bibliográficas}

BONATES, E.J.L. Procedimentos empíricos para avaliação da fragmentação. In: CONGRESSO BRASILEIRO DE LAVRA A CÉU ABERTO E SUBTERRÂNEA, 2. Belo Horizonte, 2000.

CUNNINGHAN, C.V.B. Fragmentation estimations and the Kuz-Ram model - four years on. In Proceedings 2nd Int. Symp on Rock Fragmentation by Blasting, Lulea, 1987. p. 475-487. In: Hustrulid, W. Blasting Principles for Open Pit Mining. v. 1. Rotterdam: Balkema, 1999. 301p. DJORDJEVIC, N. A two-component model of blast fragmentation. In: The AusIMM Proceedings, Austrália: Brisbane, 1999, p. 9-13.

KUZNETSOV, V.M. The mean diameter of the fragments formed by blasting rock. Soviet Mining Science, 1973. p. 144-148. In:
HUSTRULID, W. Blasting Principles for Open Pit Mining. v. 1. Rotterdam: Balkema, 1999. 301p

LILLY, P.A. An empiral method of assessing rock massing blastability. LARGE OPEN PIT MINING CONFERENCE. THE AusIMM/IE AUST NEWMAN COMNINED GROUP, Austrália: 1986. p. 89-92.

MORAIS, J.L. Simulação da fragmentação dos desmontes de rochas por explosivos. Belo Horizonte: Universidade Federal de Minas Gerais, 2004. 160p. (Tese de doutorado).

PERSON, P. A., HOLMBERG, R., LEE, J. Rock blasting and explosives engineering. CRC Press Inc: Boca Raton, 1994.

SARMA, R.S. Models for assessing the blasting performance of explosives. The University of Queensland, Austrália, 1994. (PhD Thesis).

SCOTT, A., COCKER, A., DJORDJEVIC, N., HIGGINS, M., LA ROSA, D., SARMA, K., WEDMAIER, R. Open pit blast design: analysis and optimisation. Austrália: JKMRC, Queensland, 1996. 341 p.

Artigo recebido em 21/05/2004 e aprovado em 22/10/2004.

\section{Informações importantes sobre: Geologia, Mineração, Metalurgia \& Materiais e Engenharia Civil você encontra na REM.}
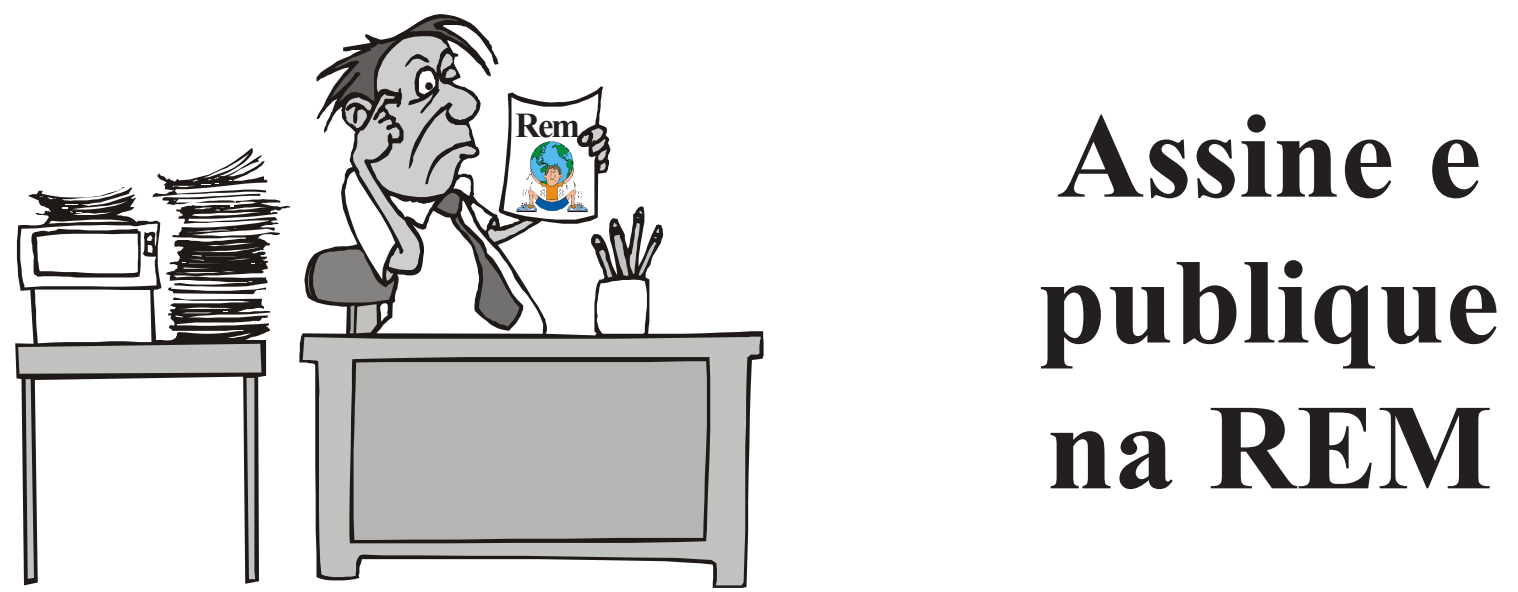

Fone/Fax: (31) 3551-4730 - E-mail: editor@rem.com.br 